\title{
Comparison of malaria treatment
} outcome of generic and innovator's anti-malarial drugs containing artemetherlumefantrine combination in the management of uncomplicated malaria amongst Tanzanian children

\author{
Manase Kilonzi ${ }^{1 *}$, Omary Minzi ${ }^{1}$, Ritah Mutagonda ${ }^{1}$, Philip Sasi ${ }^{2}$, Appolinary Kamuhabwa ${ }^{1}$ and Eleni Aklillu ${ }^{3}$
}

\begin{abstract}
Background: In 2006, artemether-lumefantrine (ALU), specifically Coartem ${ }^{\circledR}$ (Novartis Pharma AG, Basel Switzerland), was approved as the first-line drug for treatment of uncomplicated malaria in Tanzania. Due to poor availability and affordability of the innovator's product, the government of Tanzania in 2013 prequalified the use of generic antimalarial drugs, whereby Artefan ${ }^{\circledR}$ (Ajanta, Pharma Ltd, India) was the first to be approved.

Methods: This was an equivalence prospective study that aimed to determine the effectiveness of anti-malarial generic Artefan ${ }^{\circledR}$ in comparison with innovator's product Coartem ${ }^{\circledR}$. Patients aged 6 to 59 months with uncomplicated malaria were recruited and randomized to either receive Artefan ${ }^{\circledR}$ or Coartem ${ }^{\circledR}$ as a control. Participants were required to revisit clinic five times as follow up to monitor treatment outcome as per World Health Organization recommendations. On each visit, thick and thin blood smears, dried blood spot (DBS), haemoglobin concentrations and auxiliary temperature were performed and documented.
\end{abstract}

Results: Out of 230 recruited participants, 200 met inclusion criteria and were randomized equally to receive Artefan ${ }^{\circledR}$ and Coartem ${ }^{\circledR}$. The overall PCR uncorrected cure rate were $80 \%$ for Artefan ${ }^{\circledR}$ and $75 \%$ for Coartem ${ }^{\circledR}$ $(p=0.44)$. Adequate clinical and parasitological response were $82.1 \%$ for Artefan ${ }^{\circledR}$ and $74.7 \%$ for Coartem ${ }^{\circledR}$, and there was no early treatment failure (ETF) observed in both arms of treatment. Both drugs showed excellent early parasite clearance, whereby no participants had peripheral parasitaemia on day 3. Late clinical failures (LCF) were 3.6\% for Artefan $^{\circledR}$ and $1.3 \%$ for Coartem ${ }^{\circledR}(p=0.31)$, and late parasitological failure (LPF) were $15.4 \%$ for Artefan ${ }^{\circledR}$ and $22.7 \%$ for Coartem ${ }^{\circledR}(p=0.32)$. Mean haemoglobin $(g / d)$ concentrations observed on day 28 were higher compared to day 0 for both drugs, although not statistically significant. Only one (1.3\%) participant on Artefan ${ }^{\circledR}$ had temperature $\geq 37.5^{\circ} \mathrm{C}$ on day 3 .

Conclusion: The findings of this study indicate that both $\operatorname{Artefan}^{\circledR}$ and $\mathrm{Coartem}^{\circledR}$ are equivalent and effective in the management of uncomplicated malaria amongst children in the Coast part of Tanzania.

Keywords: Artefan $^{\circledR}$, Coartem $^{\circledR}$, Effectiveness

\footnotetext{
*Correspondence: manasekilonzi@yahoo.com

${ }^{1}$ Department of Clinical Pharmacy and Pharmacology, School

of Pharmacy, Muhimbili University of Health and Allied Sciences, P. O. BOX

65013, Dar es Salaam, Tanzania

Full list of author information is available at the end of the article
}

(c) The Author(s) 2019. This article is distributed under the terms of the Creative Commons Attribution 4.0 International License (http://creativecommons.org/licenses/by/4.0/), which permits unrestricted use, distribution, and reproduction in any medium, provided you give appropriate credit to the original author(s) and the source, provide a link to the Creative Commons license, and indicate if changes were made. The Creative Commons Public Domain Dedication waiver (http://creativecommons.org/ publicdomain/zero/1.0/) applies to the data made available in this article, unless otherwise stated. 


\section{Background}

Malaria continues to be one of the major public health problems in many regions of the world, particularly the developing countries [1]. African region accounts for about $90 \%$ of global malaria cases and deaths, in which majority are from Sub-Saharan African (SSA) countries [2] (carries about $80 \%$ of the global malaria burden).

A majority (90\%) of Tanzanians (both mainland and Zanzibar) live in moderate to high malaria endemic region [2]. About 10 to 12 million people in Tanzania contact malaria every year and 80,000 of them die, in majority children. Anopheles gambiae and Anopheles arabiensis are the two species of Anopheles mainly responsible for malaria transmission in Tanzania. Ninety-six percent of malaria in Tanzania is caused by Plasmodium falciparum and the remaining $4 \%$ due to Plasmodium malariae and Plasmodium ovale [2]. Co-existing of different species of Plasmodium determine appropriate treatment approach [3] and is one of the criteria to be considered in the development and review of guidelines.

Proper diagnosis and prompt treatment with effective anti-malarial drugs are one of the major tools in the control of malaria. The emergence of resistance of $P$. falciparum to anti-malarial drugs has led to changes of drugs from chloroquine to sulfadoxine-pyrimethamine (SP) and currently artemisinin-based combination therapy (ACT) $[4,5]$. In 2006, ACT was introduced in Tanzania and artemether-lumefantrine (ALU) was accepted as the first-line and dihydroartemisinin-piperaquine (DHA-P) as the second-line for management of uncomplicated malaria in both adult and children [6]. Since the introduction of ALU, the innovator's product Coartem ${ }^{\circledR}$ (Novartis Pharma AG, Basel Switzerland) was prequalified by the Tanzanian government and the product was available in all public health facilities. Due to high cost and poor availability of the innovator's product, the government of Tanzania prequalified the use of generic ALU, which is now more widely available in public health facilities than Coartem ${ }^{\circledR}$. Artefan ${ }^{\circledR}$ (Ajanta, Pharma Ltd, India) was the first generic drug to be prequalified by the government of Tanzania and is still available in the supply chain.

For the purpose of ensuring good performance and detection of emergence of resistance of anti-malarial drugs, especially those used as a first-line and secondline in a country, the World Health Organization (WHO) recommends frequent monitoring of their effectiveness. Early parasite clearance after initiation of ACT is considered to be an indicator of treatment outcome $[7,8]$. Peripheral parasitaemia on day 1 , day 2 and day 3 following initiation of ACT can be used to assess treatment outcome in individuals with uncomplicated malaria [4]. Peripheral parasitaemia on day 1 and 2 or parasite density on day 3 of $>25 \%$ parasite density of day 0 , is regarded as early treatment failure. Moreover, early parasite clearance is used as an indicator of $P$. falciparum resistance to artemisinin. Proportion of patients with detectable parasitaemia on day $3,72 \mathrm{~h}$ following initiation of antimalarial therapy is used to predict resistance (i.e. if $10 \%$ of the patients have peripheral parasitaemia on day 3 is regarded to be a sign of $P$. falciparum resistance to artemisinin $[5,9]$. Adequate clinical and parasitological response (cure rate) is mainly defined by the absence of peripheral parasitaemia on day 28.

Generic artemisinin-based combinations play a great role in the management and control of malaria in developing countries as they are highly affordable and readily available. However, availability of substandard anti-malarial drugs in SSA, including Tanzania, has been reported and they pose a threat to the gained successes in malaria control $[7,8]$. Poor quality anti-malarial drugs expose parasites to a sub-therapeutic drug pressure, thus providing windows for parasite selection, treatment failures and spread of tolerance/resistance and may also be a threat to patients' safety $[8,10]$. In 2012, Minzi et al. reported compliance of bioequivalence criteria of Artefan and Coartem in relation to AUC and Cmax, as per FDA recommendations but the generic product could not meet the 95\% confidence interval bioequivalence criterion leaving a room of doubt on the effectiveness of the generic product [11]. The objective of this study was to compare malaria treatment outcome of Artefan and Coartem in the management of uncomplicated malaria amongst Tanzanian children.

\section{Methods}

\section{Study design, site and population}

This was an equivalence prospective study that aimed to determine the effectiveness of anti-malarial generic Artefan in comparison with innovator's product Coartem. The study was conducted at Kibiti health centre in Kibiti district, which is an area in the coastal region of Tanzania, with a malaria prevalence of about $10.2 \%$. In this surveillance of anti-malarial drugs, a formula for sample size calculation in equivalence studies [12] was used to obtain the estimated sample size. One side type 1 error of $2.5 \%$ (confidence level of $95 \%$ ), power of $90 \%$, equivalence limit of 0.55 and a maximum of $10 \%$ difference of cure rates between treatment groups was considered equivalent. A minimum sample size of 93 was required for each treatment arm. In addition, a 20\% loss to follow was added, thus making the sample size of 116 participants for each treatment arm.

Consecutive sampling technique was used to enroll patients aged between 6 and 59 months with uncomplicated malaria (screening for malaria positivity was done 
by malaria Rapid Diagnostic Tests (RDT) after being examined clinically by physician). After being enrolled, patients were randomized to receive either the generic artemether-lumefantrine (Artefan) or the innovator's product (Coartem) as a control group, and thereafter followed until day-28 as per WHO guidelines [13]. Eligibility criteria were (1) aged 6-59 months, (2) uncomplicated malaria, (3) mono-infected with $P$. falciparum, (4) agreement to come to the study clinic for any febrile episode or other illnesses with the parent/guardian, (5) residence in Kibiti town perimeter, (6) absence of chronic diseases like HIV/AIDS, kidney disease and any active medical problem requiring hospitalization, and (7) provision of informed consent form signed by parents/guardians.

\section{Malaria diagnosis and treatment}

Diagnosis of malaria followed current Tanzania standard treatment guidelines (STG) in which uncomplicated malaria is defined as symptomatic malaria without signs of severity or evidence (clinical or laboratory) of vital organ dysfunction (Fever, Headache, Joint pains, Malaise, Vomiting/diarrhoea, Body ache, Body weakness, Poor appetite, Pallor, Enlarged spleen). But the clinical features listed above are not specific for malaria and can be found in several other febrile conditions. Therefore, diagnosis of uncomplicated malaria was done by using RDT (CareStart ${ }^{\mathrm{TM}}$ Malaria HRP2 (pf) (Access Bio, Ethiopia)) followed by confirmation by microscopy. Children who were malaria positive aged between 6 and 59 months were randomized to receive either Artefan or Coartem, as recommended by malaria treatment guidelines [14]. Both Artefan and Coartem are fixed combination of $20 \mathrm{mg}$ artemether and $120 \mathrm{mg}$ lumefantrine in a tablet. Study drugs used were of the same butch number and were purchased from a registered market authorization holder (MAH) in Dar es Salaam, Tanzania. Dosing was done per weight $(\mathrm{kg})$ of the patients according to the manufacturer's recommendation which was the same for both drugs. The full course of treatment for all study patients consists of 6-doses given twice daily for 3 days. The first daily dose of study was given under direct observation at the study clinic and the remaining doses were given to study participants' parents/guardians and were administered at home. Parents/guardians were asked to bring back the children on day 3, 7, 14, 21 and 28 as follow up. On day 3 parents/guardians were asked to come with the blister pack as a way of ensuring adherence to the medication. Episodes of severe malaria and recurrent malaria occurring within 14 days of therapy were treated with artesunate injection.

\section{Laboratory investigation}

Thick and thin blood smears were stained with $2 \%$ Giemsa for $30 \mathrm{~min}$ and microscopic examination was performed by trained laboratory technologists who were not involved in direct patient care. Parasite densities were calculated from thick blood smears by counting the number of asexual parasites per 200 leukocytes (or per 500 leukocytes, if the count was $<10$ asexual parasites/200 leukocytes), assuming a leukocyte count of $8000 / \mu \mathrm{l}$. A blood smear was considered negative when the examination of 100 high power fields did not reveal asexual parasites. For quality control, all slides were read by two readers. In case of discordant readings, an independent third reader was used settled discrepancies between the first and second readings. Laboratory technicians were blinded to the study participants' treatment assignments. Thin smears were used to determine the parasite species $[4,15]$.

Haemoglobin $(\mathrm{Hb})$ was determined on D0, D3, D7, D14 and D28 and quantification of $\mathrm{Hb}$ was done using a Hemocue $\mathrm{Hb} \mathrm{201}{ }^{+}$(Angelholm, Sweden) microcuvette machine, from. The drop of blood was collected in an $\mathrm{Hb} 201$ microcuvette and read using HemoCue $\mathrm{Hb} 201^{+}$ device and result was recorded in $\mathrm{g} / \mathrm{dl}$.

\section{Statistical methods}

Data were entered into an Excel sheet and analysis was done by using Statistical Package for Social Sciences (SPSS) software version 20 (SPSS Inc., Chicago, IL, USA). Mean, geometric mean, range, standard deviation and percentage were used to summarize results. Chi square was used in finding association between variables of interest. Kaplan-Meier using per-protocol approach was used to determine cure rate and malaria recurrent rate. $\mathrm{p}$-value of $<0.05$ was considered to be statistically significant.

\section{Results}

Two hundred and thirty patients aged 6-59 months with uncomplicated malaria were recruited to participate in this study. All study participants were positive on RDT and 30 patients were excluded because blood smear were found to be negative on microscopy. Two hundred patients were then randomized for treatment, whereby 100 received Artefan and another 100 Coartem. The study flowchart indicating enrolment and follow up procedures is presented in Fig. 1. Twenty-two children on Artefan and 19 on Coartem were excluded during analysis because they had co-infection of $P$. falciparum and $P$. malariae (Fig. 1). 


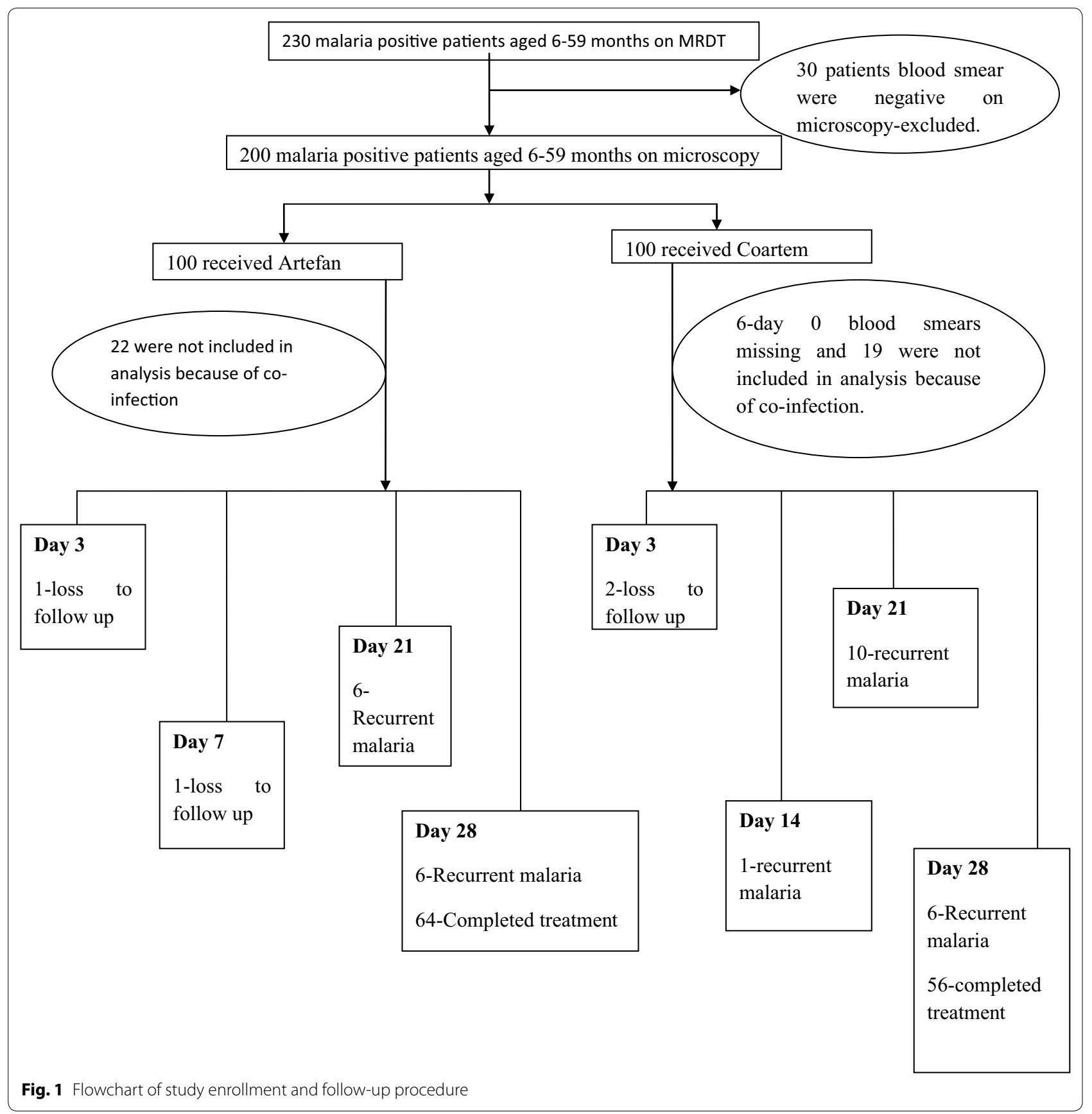

\section{Baseline characteristics of study participants}

Majority of patients were females (54\%) on Artefan and male (51.6\%) on Coartem with mean age of 33 months and 32 months, respectively. Baseline mean temperature was $38.4{ }^{\circ} \mathrm{C}$ on Artefan and $38.5^{\circ} \mathrm{C}$ on Coartem. Baseline peripheral parasitaemia geometric means were $10,074 / \mu \mathrm{l}$ in Artefan and $9320 / \mu \mathrm{l}$ in Coartem arms, while mean baseline $\mathrm{Hb}$ concentrations were $9.2 \mathrm{~g} / \mathrm{dl}$ and $9 \mathrm{~g} / \mathrm{dl}$, respectively. Before initiation of treatment, $22 \%$ of children on Artefan and $20.2 \%$ on Coartem had co-infection of $P$. falciparum and $P$. malariae. (Table 1 ). There were no significant differences in the baseline characteristics of study participants between the two study groups. By using mid upper arm circumference (MUAC), majority of patients had good nutritional status in both arms of treatment. 
Table 1 General characteristics of study participants

\begin{tabular}{|c|c|c|c|}
\hline \multirow[t]{2}{*}{ Characteristics } & \multicolumn{2}{|c|}{ Treatment arm } & \multirow[t]{2}{*}{ p-value } \\
\hline & $\begin{array}{l}\text { Artefan }^{\circledR} \\
(\mathrm{n}=78)\end{array}$ & $\begin{array}{l}\text { Coartem }^{\circledR} \\
(n=75)\end{array}$ & \\
\hline Age in mean (range) & $32.74(50-60)$ & $32.17(47-60)$ & 0.81 \\
\hline Female $n(\%)$ & $42(53.8 \%)$ & $33(44 \%)$ & 0.22 \\
\hline Temperature $\left({ }^{\circ} \mathrm{C}\right)$ in mean (SD) & $38.4(1.13)$ & $38.5(1.18)$ & 0.60 \\
\hline $\begin{array}{l}\text { Parasite density (parasite/ } \mu l) \text { in } \\
\text { mean (SD) }\end{array}$ & 9730.93 & 8745.75 & 0.16 \\
\hline Hemoglobin ( $\mathrm{g} / \mathrm{dl}$ ) in mean (SD) & $9.3(1.5)$ & $9.3(1.8)$ & 0.88 \\
\hline $\begin{array}{l}\text { Good nutritional status (based } \\
\text { on MUAC) }\end{array}$ & $71(92.2 \%)$ & $68(91.9 \%)$ & 0.94 \\
\hline
\end{tabular}

\section{Treatment outcome}

The treatment outcomes were defined as: early treatment failure (ETF) - presence of danger signs or complicated malaria with a positive blood smear on day 3 of treatment, or day 3 parasite density $>25 \%$ of day 0 parasite density, or positive blood smear on day 3 with auxiliary temperature $\geq 37.5^{\circ} \mathrm{C}$. Late clinical failure (LCF) - danger signs or severe malaria in the presence of parasitaemia on any day between day 4 and day 28 in patients who previously did not meet any of the criteria of early treatment failure, and presence of parasitaemia on any day between day 4 and day 28 with auxiliary temperature $\geq 37.5^{\circ} \mathrm{C}$ in patients who previously did not meet any of the criteria of early treatment failure. Late parasitological failure (LPF) - presence of parasitaemia on any day between day 7 and day 28 (day 42) with auxiliary temperature $<37.5^{\circ} \mathrm{C}$ in patients who previously did not meet any of the criteria of early treatment failure or late clinical failure. Adequate clinical and parasitological response (ACPR)absence of parasitaemia on day 28 irrespective of auxiliary temperature, in patients who previously did not meet any of the criteria of early treatment failure, late clinical failure or late parasitological failure.

None of the study participants had peripheral parasitaemia on day 3 on both Artefan and Coartem treatment arms. On day 3 only, 1 (1.1\%) patient on Artefan treatment arm had temperature above $37.5{ }^{\circ} \mathrm{C}$. Slightly decrease in $\mathrm{Hb}$ concentration was observed on day 3 in both arms of treatment but the difference was not statistically significant (Table 2). The mean Hb concentrations on day 28 were $10.3 \pm 1.4 \mathrm{~g} / \mathrm{dl}$ in Artefan and $10.4 \pm 1.3 \mathrm{~g} / \mathrm{dl}$ in Coartem, to some extent high compared to day 0 . No early treatment failure (ETF) was observed on day 3 on both treatment arms. Three (3.6\%) patients in Artefan and 1 (1.3\%) in Coartem treatment arms were classified as late clinical failure (LCF). Fourteen (16.7\%) patients in Artefan and 16 (21.3\%) in Coartem treatment arms were classified as late parasitological failures (LPF), most of the failures were observed
Table 2 Temperature persistence and parasite clearance

\begin{tabular}{|c|c|c|c|}
\hline \multirow[t]{2}{*}{ Characteristics } & \multicolumn{2}{|c|}{ Treatment outcome } & \multirow[t]{2}{*}{ p-value } \\
\hline & $\begin{array}{l}\text { Artefan }{ }^{\circledR} \text { arm } \\
(n=78)\end{array}$ & $\begin{array}{l}\text { Coartem }^{\circledR} \text { arm } \\
(n=75)\end{array}$ & \\
\hline \multicolumn{4}{|c|}{ Temperature persistence (temperature $\geq 37.5^{\circ} \mathrm{C}$ ) } \\
\hline On day 0 & $66(84.6 \%)$ & $61(81.3 \%)$ & 0.60 \\
\hline On day 3 & $1(1.3 \%)$ & $0(0.0 \%)$ & 0.54 \\
\hline On day 7 & $2(2.9 \%)$ & $0(0.0 \%)$ & 0.25 \\
\hline On day 14 & $2(2.9 \%)$ & $0(0.0 \%)$ & 0.26 \\
\hline On day 21 & $1(1.6 \%)$ & $0(0.0 \%)$ & 0.51 \\
\hline On day 28 & $3(5 \%)$ & $1(1.7 \%)$ & 0.31 \\
\hline \multicolumn{4}{|l|}{ Parasite clearance } \\
\hline Smear positive on day 3 & 0 & 0 & \\
\hline Smear positive on day 7 & 0 & 0 & \\
\hline Smear positive on day 14 & $0(0.0 \%)$ & $1(1.1 \%)$ & 0.71 \\
\hline Smear positive on day 21 & $6(7.7 \%)$ & $10(13.3 \%)$ & 0.42 \\
\hline Smear positive on day 28 & $6(7.7 \%)$ & $6(8 \%)$ & 0.97 \\
\hline \multicolumn{4}{|c|}{ Mean hemoglobin concentration (SD) } \\
\hline Day 3 & $8.9(1.5)$ & $8.8(1.4)$ & 0.96 \\
\hline Day 7 & $9.0(1.4)$ & $9.1(1.5)$ & 0.73 \\
\hline Day 14 & $9.8(1.3)$ & $9.6(1.3)$ & 0.43 \\
\hline Day 21 & $10(1.4)$ & $9.8(1.4)$ & 0.97 \\
\hline Day 28 & $10.3(1.4)$ & $10.4(1.3)$ & 0.74 \\
\hline
\end{tabular}

Table 3 Treatment outcome during 28 days follow up period

\begin{tabular}{|c|c|c|c|}
\hline \multirow[t]{2}{*}{ Treatment outcome } & \multicolumn{2}{|c|}{ Treatment arm } & \multirow[t]{2}{*}{ p-value } \\
\hline & $\begin{array}{l}\text { Artefan }^{\circledR} \\
(\mathrm{n}=84)\end{array}$ & $\begin{array}{l}\text { Coartem }^{\circledR} \\
(n=75)\end{array}$ & \\
\hline Late clinical failure (LCF) & $1(3.6 \%)$ & $1(1.3 \%)$ & 0.31 \\
\hline Late parasitological failure (LPF) & $12(15.4 \%)$ & $17(22.7 \%)$ & 0.32 \\
\hline $\begin{array}{l}\text { Adequate clinical and parasito- } \\
\text { logical response (ACPR) }\end{array}$ & $64(82.1 \%)$ & $56(74.7 \%)$ & 0.44 \\
\hline
\end{tabular}

on day 21 and day 28. Adequate clinical and parasitological responses (ACPR) on day 28 were $70(83.3 \%)$ patients in Artefan and $58(77.3 \%)$ in Coartem treatment arms, but the difference in ACPR was not statistically significant $(\mathrm{p}=0.34)$ between the two drugs (Table 3). Children in the Coartem (27\%) arm had high risk to get recurrent malaria compared to those in the Artefan (17\%) arm, but the difference was not statistically significant $(p=0.23)$ (Fig. 2).

\section{Discussion}

After the introduction of artemisinin-based combination therapy $(\mathrm{ACT})$ as the first-line and second-line option in the management of uncomplicated and complicated malaria by the WHO together with other methods of malaria prevention such as insecticide-treated nets (ITN) 


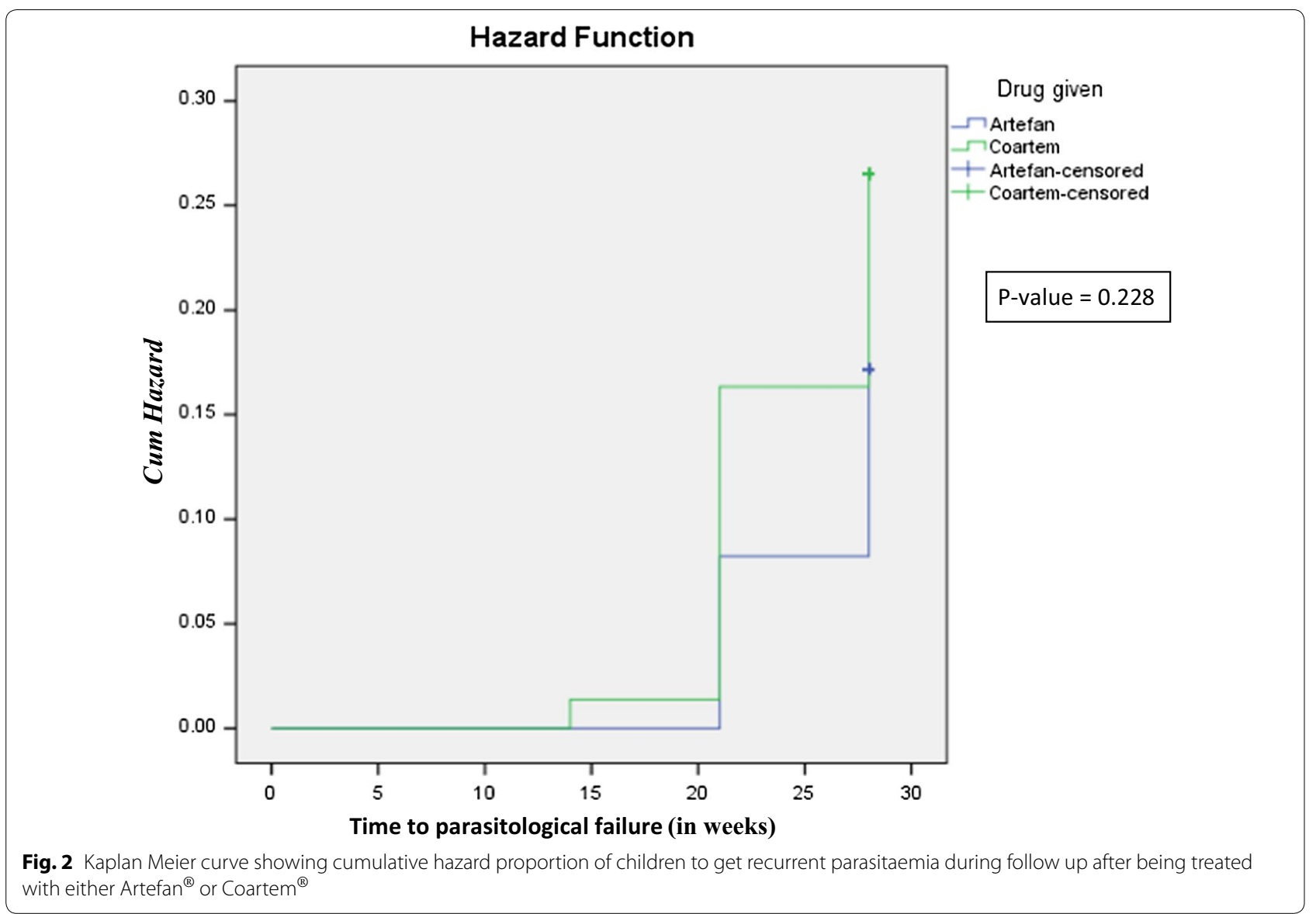

and indoor spraying, prevalence of malaria has decline in many countries. Protecting the efficacy of ACT for the treatment for falciparum malaria is among the top global public health priorities. The presence of falsified and substandard drugs has been reported in many parts of the world, including sub-Saharan Africa (SSA) [16]. The WHO has identified anti-malarials as one group of drugs at highest risk of being falsified or sub-standard, because of high demand especially in moderate to high endemic regions. Previous studies reported the presence of poor quality and substandard anti-malarial drugs in the Tanzanian market $[17,18]$. Regular surveillance and assessment of the performance of generic anti-malarials through therapeutic efficacy studies is of importance for malaria control.

In this study, the therapeutic efficacy of generic antimalarial Artefan and innovator's product Coartem were compared. Early parasite clearance was excellent for both drugs with no detected peripheral parasitaemia on day 3 (72 h, following initiation of therapy) using microscopy. Temperature clearance was also outstanding for both Artefan and Coartem, with only $1(1.1 \%)$ patient in the Artefan arm having temperature above $37.5^{\circ} \mathrm{C}$ on day 3 .
The early parasite clearance in this study on day 3 was the same as what was reported in Uganda, in which early parasite clearance following treatment with artemetherlumefantrine was excellent [4].

Resistance of Plasmodium species to artemisinin has been reported in eastern and southern Asian countries [19], but not yet in Africa. In the present study, none of the study participants treated with Artefan or Coartem had peripheral parasitaemia on day 3. This may indicate absence of resistant strains of $P$. falciparum to artemisinin in Tanzania. This is in line with the WHO 2009 anti-malarial protocol, that if $10 \%$ of the study participants have peripheral parasitaemia on day 3 , it is an indicator of emergence of artemisinin resistance to Plasmodium species [13].

Early treatment failure (ETF) was not observed in both Artefan and Coartem treatment arms, whereas late clinical failures (LCF) were $3.6 \%$ and $1.3 \%$ in Artefan and Coartem arms, respectively. However, late parasitological failures (LPF) were high in both Artefan (15.4\%) and Coartem (22.7\%) arms. Artemisinin products (for this study artemether) have a short half-life. Since most of the LCF and LPF occurred on day 21 and 28 with only 
one LPF occurring on day 14, its means the partner drug (lumefantrine) offers a prolonged protection against malaria of two to 3 weeks. From the survival analysis, cumulative hazard proportion curve shows that children using Coartem might be at higher risk of parasitological failure compared to children using Artefan. The observed differences in late treatment failures (LCF and LPF) between Artefan and Coartem were not statistically significant, an indication that the generic anti-malarial Artefan is effective for management of uncomplicated malaria in Tanzanian children.

The observed LCF in this study is comparable to what was reported in Ethiopia [20]. In the Ethiopian study, LCF were $1.4 \%$ on day 21 and $2.8 \%$ on day 28 . On the other hand, the results of LCF are less compared to $17.8 \%$ which was in Uganda [4]. Moreover, late parasitological failures observed in this study are higher compared to what was reported in Ethiopia by Kinfu et al. and Mekonnen et al., in which LPF of $0.0 \%$ and $4.5 \%$, respectively, were observed after 28 days follow following treatment with ALU [20, 21]. In addition, the observed LPF is much lower compared 32.9\% that was reported in Uganda [4]. The observed differences in these studies could be due to endemicity of malaria in the respective study areas and nature of the study. Most of the studies were efficacy studies in which community health workers were deployed work with parents and guardians in making sure that all children take their medicines as prescribed.

The WHO recommends the use of microscopy as a golden standard for diagnosis of malaria in middle and low income countries. In this study, microscopy was also used to assess the performance of anti-malarial drugs. Using microscopy, the observed adequate clinical and parasitological response (ACPR) were $83.3 \%$ in children in the Artefan and $77.3 \%$ in the Coartem arms. PCR uncorrected cure rate were $>80 \%$ for Artefan and $75 \%$ for Coartem. These results indicate that both generic Artefan and innovator's product Coartem are effective for the treatment of uncomplicated malaria in children.

High adequate clinical and parasitological responses and malaria cure rate of ALU have been reported in different therapeutic efficacy studies. For instance, 100\% adequate clinical and parasitological response were reported in South West Ethiopia in children under 5 years of age (23), and very low (45.4\%) in Uganda [4]. PCR uncorrected cure rate were also reported to be very high $(98.8 \%)$ in northwest Ethiopia [22] and south west Ethiopia (96.3\%) [23]. The ability of artemisinin to clear the biomass of Plasmodium within short hours of treatment and prevention of maturation of the gametocytes by the partner drug (lumefantrine) offer the maximum performance of ALU.
Based on mid upper arm circumference (MUAC), most of the participants in this study had good nutrition status. The difference in clearing malaria parasites between those with poor nutrition status and good nutrition status could not be established. Studies have shown conflicting conclusions on the role of nutrition in relation to malaria parasite clearance with some linking poor parasite clearance and malaria morbidity and mortality with poor nutrition status $[24,25]$, while other studies reporting negative association between parasite clearance with malaria-induced morbidity and mortality with poor nutrition status [26, 27]. Nevertheless, emphasis must be put on taking food stuffs which are rich in iron in order to minimize incidences of anaemia in children suffering from malaria [28].

The limitation of this study is that it was designed to mimic the routine standard of care for management of uncomplicated malaria in Tanzania. In this approach, patients are given anti-malarial drugs with instruction to take the prescribed doses at home. Therefore, information on the parasite density during day 1 and day 2 after drug administration were not collected. In addition, adherence to the prescribed drugs was based on self-reports from patients, parents or guardians. Also, microscopy instead of molecular techniques, such PCR, was used to identify Plasmodium species. Therefore, the reported cure rate in both arms of the study are PCR uncorrected.

\section{Conclusion}

This study revealed that Artefan as generic anti-malarial drug containing artemether and lumefantrine is equivalent to the innovator's product Coartem in the management of uncomplicated malaria in Coast region of Tanzania. Due to low cost and availability, the use of Artefan as the generic drug is advantageous in comparison to the relatively expensive innovator's drug such as Coartem. Therefore, the findings of this study support continuing use of generic anti-malarial drugs in the management of uncomplicated malaria in children.

\footnotetext{
Authors' contributions

MK, OM, AK and EA conceived and deigned the study. MK participated in data collection. MK, RM and SP participated in data analysis. All authors participated in developing the manuscript. All authors read and approved the final manuscript.

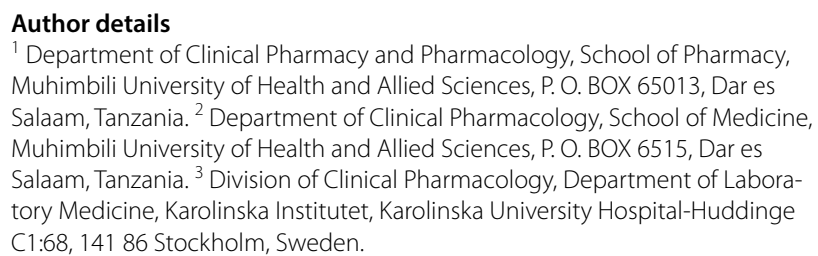




\begin{abstract}
Acknowledgements
We thank the Swedish research agency for developing countries (Sida) through Malaria and Neglected Tropical Diseases Subprogram at Muhimbili University of Health and Allied Sciences for providing financial support for this study. The authors thank the parents and guardians of the children and the district medical and administrative leaders in Kibiti for supporting the study. We also grateful to the research assistants that gave support during recruitment of study participants, treatment, collection of blood samples and analysis at the study site.
\end{abstract}

\section{Competing interests}

The authors declare that they have no competing interests.

\section{Availability of data and materials}

The datasets used and/or analysed during the current study are available from the corresponding author on reasonable request.

\section{Consent for publication}

Not applicable.

\section{Ethics approval and consent to participate}

The study protocol was reviewed and approved by Research and Publication committee of Muhimbili University of Health and Allied Sciences and permission was obtained from Kibiti district council. Informed consent forms signed by parents or guardians of study participants were obtained before commencing the study. For confidentiality purposes, each participant was assigned with an identity number instead of his/her name.

\section{Funding}

This study was funded by Sida through Malaria and Neglected Tropical Diseases Subprogram at Muhimbili University of Health and Allied Sciences. Sida-MUHAS Malaria and NTD subprogram.

\section{Publisher's Note}

Springer Nature remains neutral with regard to jurisdictional claims in published maps and institutional affiliations.

Received: 10 October 2018 Accepted: 5 April 2019

Published online: 11 April 2019

\section{References}

1. WHO. World malaria report 2017. Geneva: World Health Organization; 2017.

2. USAID, CDC. U. S. President's Malaria Initiative, Tanzania malaria operational plan 2017. Washington: USAID; 2017. p. 1-45.

3. Barber BE, William T, Grigg MJ, Yeo TW, Anstey NM. Limitations of microscopy to differentiate Plasmodium species in a region co-endemic for Plasmodium falciparum, Plasmodium vivax and Plasmodium knowlesi. Malar J. 2013;12:8.

4. Muhindo MK, Kakuru A, Jagannathan P, Talisuna A, Osilo E, Orukan F, et al. Early parasite clearance following artemisinin-based combination therapy among Ugandan children with uncomplicated Plasmodium falciparum malaria. Malar J. 2014;13:32.

5. Stepniewska K, Ashley E, Lee SJ, Anstey N, Karen I, Binh TQ, et al. In vivo parasitological measures of artemisinin susceptibility. J Infect Dis. 2015;201:570-9.

6. Social welfare, NMCP-National Malaria Control Programme. National guidelines for diagnosis and treatment of malaria. Dar es Salaam; 2006.

7. Verduin-Muttiganzi R, Verduin-Muttiganzi G. Assessment of the incidence of substandard drugs in developing countries. Trop Med Int Health. 1998;3:602.

8. Atemnkeng MA, De Cock K, Plaizier-Vercammen J. Quality control of active ingredients in artemisinin-derivative antimalarials within Kenya and DR Congo. Trop Med Int Health. 2007;12:68-74.
9. Flegg JA, Guerin PJ, White NJ, Stepniewska K. Standardizing the measurement of parasite clearance in falciparum malaria: the parasite clearance estimator. Malar J. 2011;10:339.

10. Newton PN, McGready R, Fernandez F, Green MD, Sunjio M, Bruneton C, et al. Manslaughter by fake artesunate in Asia — will Africa be next? PLoS Med. 2006;3:e197.

11. Minzi OMS, Marealle IA, Shekalaghe S, Juma O, Ngaimisi E, Chemba M, et al. Comparison of bioavailability between the most available generic tablet formulation containing artemether and lumefantrine on the Tanzanian market and the innovator's product. Malar J. 2013;12:174.

12. Flight $L$, Julious $S A$. Practical guide to sample size calculations: noninferiority and equivalence trials. Pharm Stat. 2016;15:80-9.

13. Ho W. Methods for surveillance of antimalarial drug efficacy. Geneva: World Health Organization; 2009. p. 90.

14. Ministry of Health, Tanzania. Standard treatment guidelines and national essential medicines list, Tanzania mainland. Dar es Salaam: Ministry of Health; 2017. p. 36

15. WHO. Assessment and monitoring of antimalarial drug efficacy for the treatment of uncomplicated falciparum malaria. Geneva: World Health Organization; 2003.

16. Baird JK. Effectiveness of antimalarial drugs. N Engl J Med. 2005:352:1565-77.

17. Minzi OM, Moshi MJ, Hipolite D, Massele AY, Tomson G, Ericsson O, et al. Evaluation of the quality of amodiaquine and sulphadoxine/pyrimethamine tablets sold by private wholesale pharmacies in Dar Es Salaam Tanzania. J Clin Pharm Ther. 2003;28:117-22.

18. Hebron Y, Tettey JNA, Pournamdari M, Watson DG. The chemical and pharmaceutical equivalence of sulphadoxine/pyrimethamine tablets sold on the Tanzanian market. J Clin Pharm Ther. 2005;30:575-81.

19. Noedl H, Se Y, Sriwichai S, Schaecher K, Teja-isavadharm P, Smith B, et al. Artemisinin resistance in Cambodia: a clinical trial designed to address an emerging problem in Southeast Asia. Clin Infect Dis. 2010;51:e82-9.

20. Mekonnen SK, Medhin G, Berhe N, Clouse RM, Aseffa A. Efficacy of artemether-lumefantrine therapy for the treatment of uncomplicated Plasmodium falciparum malaria in Southwestern Ethiopia. Malar J. 2015:14:317.

21. Kinfu G, Gebre-Selassie S, Fikrie N. Therapeutic efficacy of artemetherlumefantrine for the treatment of uncomplicated Plasmodium falciparum malaria in Northern Ethiopia. Malar Res Treat. 2012;2012:548710.

22. Teklemariam M, Assefa A, Kassa M, Mohammed H, Mamo H. Therapeutic efficacy of artemether-lumefantrine against uncomplicated Plasmodium falciparum malaria in a high-transmission area in northwest Ethiopia. PLOS ONE. 2017;12:e0176004.

23. Assefa A, Kassa M, Tadese G, Mohamed H, Animut A, Mengesha T. Therapeutic efficacy of artemether/lumefantrine $\left(\right.$ Coartem $\left.^{\circledR}\right)$ against Plasmodium falciparum in Kersa, South West Ethiopia. Parasit Vectors. 2010;3:1.

24. Shankar AH. Nutritional modulation of malaria morbidity and mortality. J Infect Dis. 2000;182:S37-53.

25. Genton B, AI-Yaman F, Ginny M, Taraika J, Alpers MP. Relation of anthropometry to malaria morbidity and immunity in Papua New Guinean children. Am J Clin Nutr. 1998:68:734-41.

26. Scrimshaw NS. Symposium: nutrition and infection, prologue and progress since 1968. Historical concepts of interactions, synergism and antagonism between nutrition and infection. J Nutr. 1968;2003(133):316-21.

27. Almeida M, Alexandre A, Benzecry SG, Siqueira M, Vitor-silva S, Melo GC. The association between nutritional status and malaria in children from a rural community in the Amazonian region: a longitudinal study. PLoS Negl Trop Dis. 2015;9:e0003743.

28. Kotepui M, Phunphuech B, Phiwklam N, Chupeerach C, Duangmano S. Effect of malarial infection on haematological parameters in population near Thailand-Myanmar border. Malar J. 2014;13:218. 